\title{
Serial OCT Imaging of Retina in HELLP Syndrome
}

\section{Tony Y Chen and Neelakshi Bhagat*}

Institute of Ophthalmology and Visual Science, Rutgers-New Jersey Medical School, Newark, New Jersey, United States

*Corresponding Author: Neelakshi Bhagat, Institute of Ophthalmology and Visual Science, Rutgers-New Jersey Medical School, Newark, New Jersey, United States.
Received: May 22, 2020

Published: June 10, 2020

(C) All rights are reserved by Tony Y Chen and Neelakshi Bhagat.

\section{Abstract}

Exudative retinal detachment (ERD) is a rare ophthalmic complication of eclampsia and HELLP syndrome. We report a case of acute painless severe vision loss in a multigravida pregnant woman with HELLP syndrome caused by bilateral ERDs. The use of systemic corticosteroids, and meticulous blood pressure management, resulted in rapid resolution of subretinal fluid and visual recovery. Disruptions in ellipsoid zone and retinal pigmented epithelium, however, persisted for months after resolution of subretinal fluid.

Keywords: Exudative Retinal Detachment; Serous Retinal Detachment; Pre-eclampsia; HELLP Syndrome; Corticosteroids

\section{Abbreviations}

FA: Fluorescein Angiogram; FAF: Fundus Autofluorescence; HELLP: Hemolysis, Elevated Liver Enzyme, Low Platelet; ERD: Exudative Retinal Detachment; EZ: Ellipsoid Zone; RPE: Retinal Pigmented Epithelium; SD-OCT: Spectral Domain Optical Coherence Tomography; SRF: Subretinal Fluid

\section{Introduction}

Exudative retinal detachment (ERD) is a rare ophthalmic complication of pre-eclampsia [1]. The risk of exudative detachment markedly increases from a reported incidence of $1 \%$ in pre-eclampsia to $10 \%$ in eclampsia [2]. Pre-eclamptic/eclamptic patients with HELLP syndrome (Hemolysis, Elevated Liver Enzyme, Low Platelet) are estimated to be 7 times more likely to develop ERD, although its incidence is not well-established [3]. Most serous retinal detachments spontaneously resolve with time. In this report we describe a case of bilateral ERD in a patient with pre-eclampsia and HELLP syndrome managed with systemic corticosteroids. Serial ophthalmic imaging demonstrates persistent disruptions in ellipsoid zone (EZ) and retinal pigmented epithelium (RPE) for weeks after clinical resolution of bilateral ERDs.

\section{Case Presentation}

A 33-year old multigravida woman with limited prenatal care presented with rupture of membranes (amniotic sac), acute onset of severe headache, and bilateral vision loss at 39 weeks of gestation. She was a cystic fibrosis carrier and her past medical history was significant for gestational diabetes in her previous pregnancy. Her blood pressure was 173/108 mmHg on presentation. She had placental abruption and had vaginal delivery of her demised fetus. Laboratory evaluation showed LDH of $1114 \mathrm{U} / \mathrm{L}$, AST of 173 $\mathrm{U} / \mathrm{L}, \mathrm{ALT}$ of $26 \mathrm{U} / \mathrm{L}$, platelet count of $58 \times 10^{9} / \mathrm{L}$ and a spot urine protein of $100 \mathrm{mg} / \mathrm{dL}$, consistent with pre-eclampsia with HELLP syndrome. A computed tomography of head without contrast was performed which showed no acute intracranial abnormality. Ophthalmology was consulted after delivery for evaluation of persistent bilateral decreased vision. Initial visual acuity was count fingers in both eyes. There was no afferent pupillary defect. Anterior segment examination and intraocular pressures of both eyes were normal. Dilated fundus examination revealed bilateral bullous ERD in both eyes. She was started on intravenous methylprednisolone $250 \mathrm{mg}$ twice a day and blood pressure were normalized with antihypertensives. Within 24 hours, the vision improved to 20/200 and $20 / 80$ in right and left eyes, respectively; most of the subretinal fluid (SRF) had resolved and remained only in inferior periphery of both eyes. Multiple discrete, yellow fibrinous subretinal deposits were noted in the posterior pole as the SRF resolved, mainly in the temporal macula (Figure 1A). Fundus autofluorescence (FAF) of the posterior pole showed areas of discrete fluorescence corresponding to the subretinal deposits (Figure 1B). On spectral domain optical coherence tomography (SD-OCT), shallow areas of subretinal fluid interspersed with subretinal hyperreflective materials were seen in the macula (Figure 1C). Early frames of fluorescein angio- 
gram (FA) showed delayed and poor perfusion of choriocapillaris in posterior pole (Figure 2A), with multifocal hyperfluorescent defects that leaked in late frames (Figure 2B).
On follow-up examination two days later, patient had rapid improvement of her visual acuity to 20/50 and 20/40 in right and left eye, respectively, with further clinical resolution of SRF. (Figure

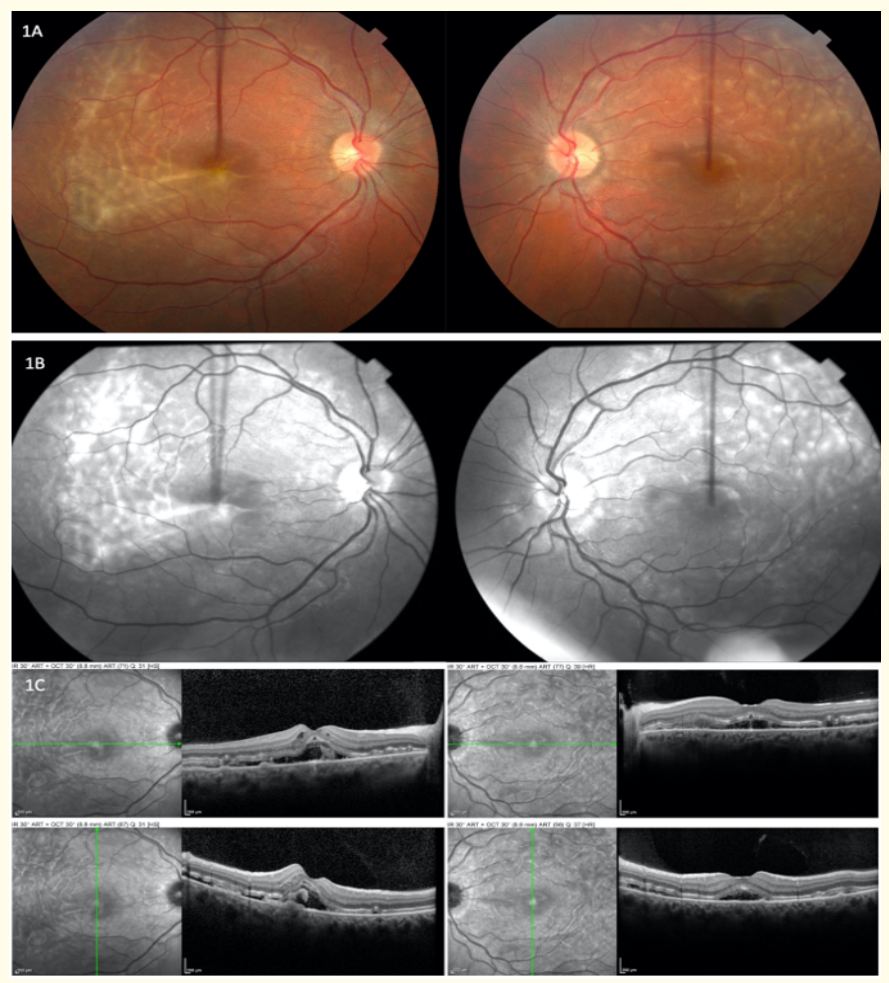

Figure 1: Day 2 after initial presentation of severe vision loss due to extensive exudative retinal detachment. Fundus photograph shows minimal subretinal fluid in the posterior pole with discrete, yellow fibrinous subretinal deposits (1A). FAF reveals fluorescence corresponding to the subretinal deposits (1B). Small amount of subretinal fluid with interspersed hyperreflective material is seen in the perimacular area on SD-OCT (1C).

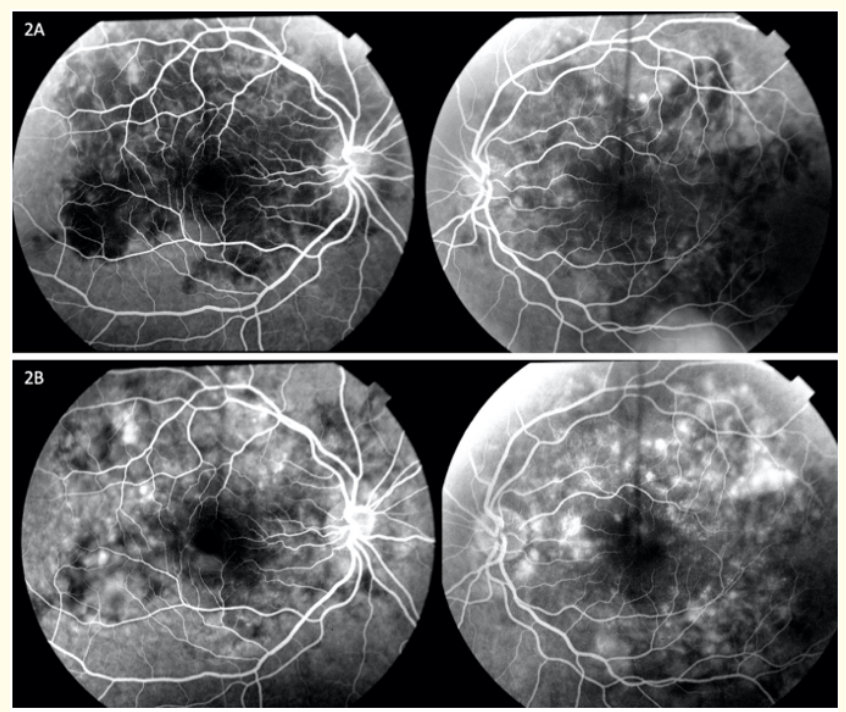

Figure 2: Day 2 after initial presentation. Fluorescein Angiogram (FA): Early frames show delayed and poor perfusion of choriocapillaris in the posterior poles (2A). Multiple hyperfluorescent leaks in late frames (2B). 
3A). Repeat FA showed patchy but improved choroidal perfusion with mottled hyperfluorescence (Figure 3B). Serial SD-OCT at 2 days (Figure 4A), 4 days (Figure 4B), and 3.5 weeks (Figure 4C) revealed complete resolution of SRF. At 2-month follow-up visit, her visual acuity was stable at 20/30 and 20/20. Dilated examination showed areas of subtle hypopigmentation in temporal macula (Figure $5 \mathrm{~A}$ ), with multiple small round autofluorescent lesions on FAF (Figure 5B). Areas of EZ/RPE disruption with interspersed subretinal deposits can be seen on SD-OCT (Figure 5C).

\section{Discussion}

We present a case of acute, bilateral ERDs in a pre-eclamptic patient with HELLP syndrome. Microthrombi related to disseminated intravascular coagulation in HELLP syndrome can cause blockage and poor blood flow through choriocapillaris. Choroidal hyperpermeability associated with severe choroidal ischemia leads to serous retinal detachments [4]. During the acute phase of visual loss, FA showed a delay of choroidal flush and choroidal nonperfusion which improved quickly, as seen on repeat FA, within 2 days of hy-

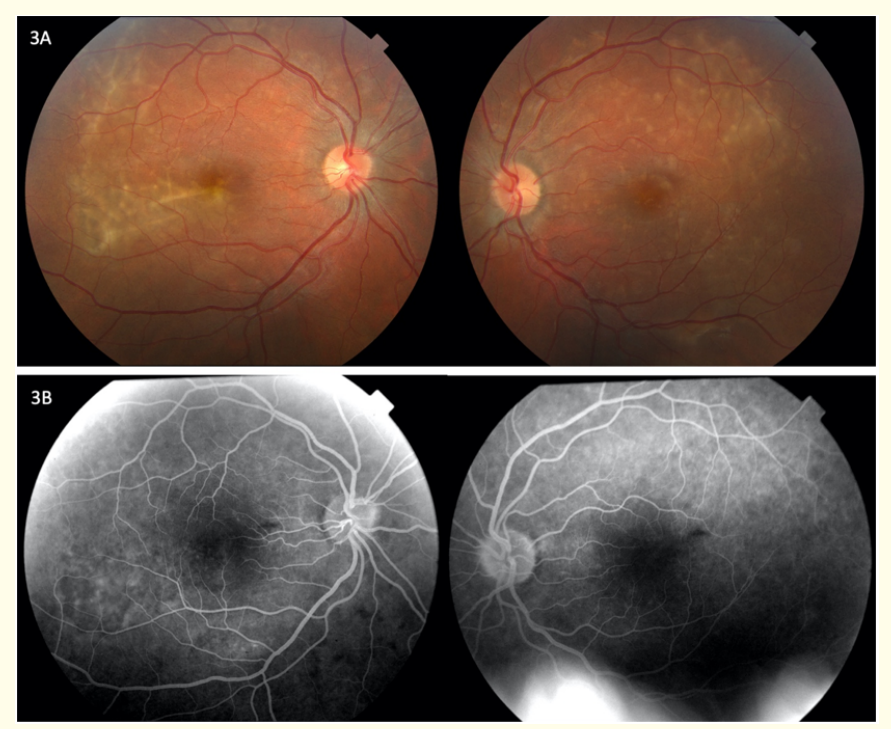

Figure 3: Day 4 after initial presentation. Fundus photo (3A). FA shows patchy but improved choroidal perfusion (3B) compared to the Day 2 FA shown in figure 2.
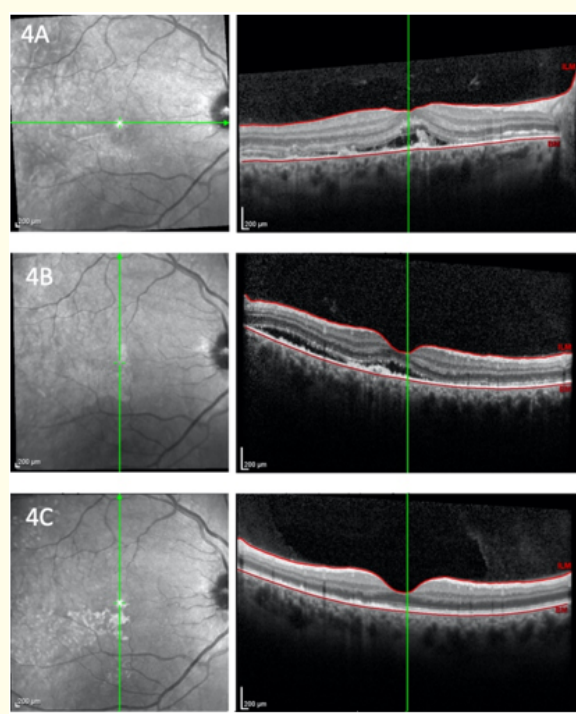
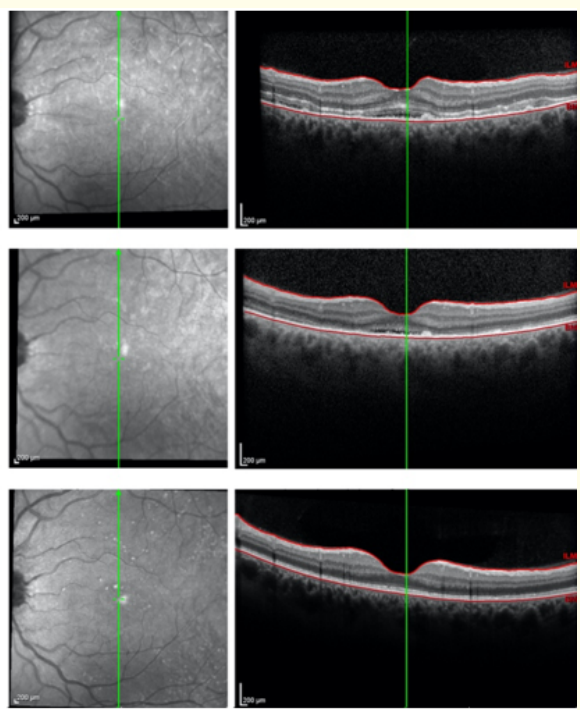

Figure 4: Serial SD-OCTs demonstrate progressive improvement of subretinal fluid on day 2 (4A), day 4 (4B), and complete resolution of subretinal fluid at week 3.5 (4C). 

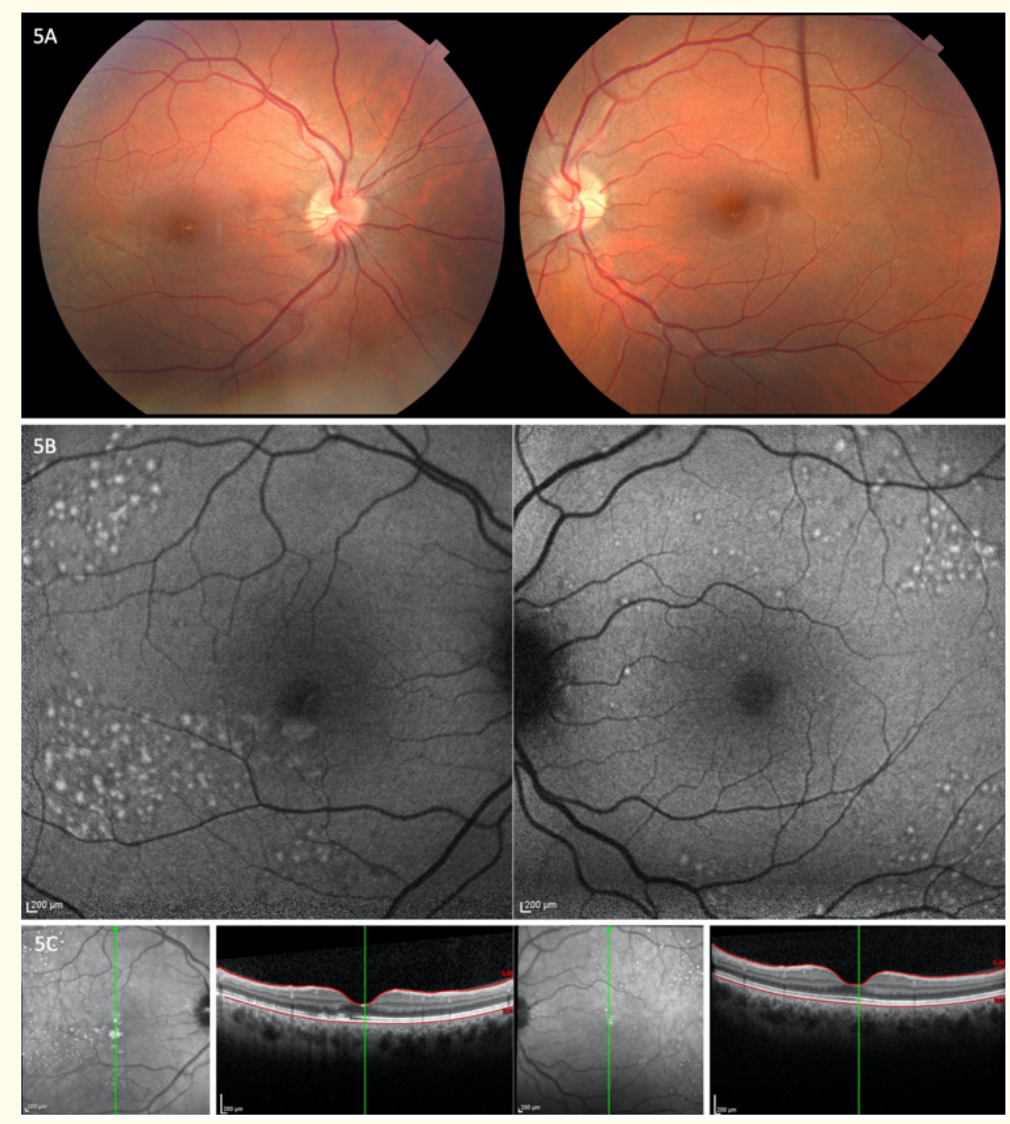

Figure 5: Fundus photograph, FAF, and SD-OCT at 2-month follow-up visit shows complete resolution of SRF. Subtle hypopigmentation can be seen in temporal macula (5A) corresponding to the autofluorescent lesions on FAF (5B).

Persistent EZ/RPE disruptions are seen on SD-OCT (5C).

pertension management and use of systemic corticosteroids. Prior studies have suggested that choroidal ischemia results from elevated blood pressure, along with endothelial dysfunction vasospasm, oxidative stress, and hemolysis associated with pre-eclampsia $[3,4]$. Treatment for ERD in the setting of pre-eclampsia/eclampsia with or without HELLP syndrome remains controversial to date. Most cases spontaneously improve after delivery when blood pressure is controlled, and medical condition stabilizes. Systemic corticosteroid may be helpful in the management of ERD although it is not well-established [5-7]. Use of corticosteroids and meticulous management of blood pressure in our case led to marked resolution of bullous ERD within a day, although the discrete subretinal fibrinous deposits and perimacular minimal SRF took 3 weeks to resolve completely as seen on the serial OCTs. Subretinal fibrinous exudates and EZ/RPE disruptions continued to be seen on SD-OCT beyond complete resolution of SRF. These outer retinal and RPE findings may represent permanent sequelae.

\section{Conclusion}

ERD is a rare complication of pre-eclampsia, especially in those with HELLP syndrome. Subretinal fibrinous deposits may be seen in severe cases but spontaneous quick recovery of ERDs with visual improvement can be achieved with use of systemic corticosteroid. Despite rapid resolution of SRF within days in this condition, longterm sequelae of ischemic choriocapillaris can be seen in outer retinal and RPE layers.

\section{Bibliography}

1. Schultz Kara L., et al. "Ocular Disease in Pregnancy". Current Opinion in Ophthalmology 16.5 (2005): 308-314.

2. Schechet Sid and Sunness Janet S. "Pregnancy-Related Diseases". Ryan's Retina, Sixth edition, edited by Andrew Schechet, Elsevier (2018): 95, 1771-1785. 
3. Vigil-De Gracia Paulino and Luis Ortega-Paz. "Retinal Detachment in Association with Pre-Eclampsia, Eclampsia, and HELLP Syndrome". International Journal of Gynecology and Obstetrics 114.3 (2011): 223-225.

4. Lee Christopher Seungkyu., et al. "Serous retinal detachment in preeclampsia and malignant hypertension". Eye (London, England) 33.11 (2019): 1707-1714.

5. Schönfeld CL. "Bilateral Exudative Retinal Detachment in HELLP Syndrome". Case Reports in Ophthalmology 3.1 (2012): 35-37.

6. Chakraborti Chandana., et al. "A rare case of bilateral total retinal detachment in pre-eclampsic primigravida patient". Sudanese Journal of Ophthalmology 8.2 (2016): 62-64.

7. Srećković Sunčica B., et al. "Bilateral retinal detachment in a case of preeclampsia". Bosnian Journal of Basic Medical Sciences 11.2 (2011): 129-131.

\section{Assets from publication with us}

- Prompt Acknowledgement after receiving the article

- Thorough Double blinded peer review

- Rapid Publication

- Issue of Publication Certificate

- High visibility of your Published work

Website: www.actascientific.com/

Submit Article: www.actascientific.com/submission.php

Email us: editor@actascientific.com

Contact us: +919182824667 